\title{
Radial deformation and stability of single-wall carbon nanotubes under hydrostatic pressure
}

\author{
Masayuki Hasegawa* and Kazume Nishidate \\ Faculty of Engineering, Iwate University, Morioka 020-8551, Japan \\ (Received 14 March 2006; revised manuscript received 30 June 2006; published 5 September 2006)
}

\begin{abstract}
In this paper we have developed a theory of energetics for isolated single-wall carbon nanotubes (SWNTs) deformed in the radial direction, and applied this theory to investigate their deformation characteristics and stability under hydrostatic pressure. The starting point of the theory is the strain energy of SWNTs predicted by $a b$ initio calculations based on the density functional theory (DFT), which shows the same behavior as that obtained for the continuum elastic shell model. We extend this result for inflated SWNTs with circular cross section to calculate the deformation energy of a deformed SWNT without performing further DFT calculations. This extension is then complemented by a van der Waals interaction, which is not fully taken into account in the DFT approximations currently in use but becomes important in highly deformed tubes. We find that the minimum pressure, $P_{1}$, for the radial deformation to occur is proportional to the inverse cube of tube diameter, $D$, in agreement with the recent theoretical predictions as well as the classical theory of buckling. The radial deformation of SWNTs with $D<2.5 \mathrm{~nm}$ is found to be elastic up to very high pressure and they hardly collapse. On the other hand, SWNTs with $D>2.5 \mathrm{~nm}$ show a plastic deformation and collapse if the applied hydrostatic pressure exceeds a critical value, which is about $30-40 \%$ higher than $P_{1}$ and also varies as $D^{-3}$ though approximately. These SWNTs with large $D$ collapse when the cross-sectional area is about $60 \%$ reduced with respect to the circular one. It is also found that for SWNTs with $D>7.0 \mathrm{~nm}$, the plastically deformed (collapsed) state is more stable than the inflated one. This critical value of $D$ is somewhat larger than previously predicted.
\end{abstract}

DOI: 10.1103/PhysRevB.74.115401 PACS number(s): 61.46. $-\mathrm{w}$, 61.48.+c, 81.40.Lm, 81.40.Vw

\section{INTRODUCTION}

Since the discovery of carbon nanotubes, ${ }^{1}$ extensive experimental and theoretical studies have been made for their structural, mechanical, thermodynamic, and electronic properties. ${ }^{2}$ These studies have been motivated by scientific and technological interest in their amazing properties due to the exceptional nanostructure unparalleled by other materials. In particular, carbon nanotubes show extraordinarily high stiffness and strength in the axial direction and enormous flexibility for bending. These unusual mechanical properties can be explained by the strong in-plane C-C covalent bond and have implied that carbon nanotubes are natural candidates for nanoscale fibers in the strong, flexible, and lightweight composite materials. The axial Young's modulus of carbon nanotubes, both experimental ${ }^{3-5}$ and theoretical, ${ }^{6-10}$ shows some scatter (see the compilation in Ref. 10) but its value of $\sim 1 \mathrm{TPa}$ for both single-wall nanotubes (SWNTs) and multiwall nanotubes (MWNTs) is now commonly accepted. This scatter partly arises from the difficulty in modeling nanoscale tubes in terms of an effective wall thickness. ${ }^{11,12}$ On the other hand, carbon nanotube bundles and crystals have been the main focus in the studies of the radial elastic properties and their bulk modulus of $30-40 \mathrm{GPa}^{13-16}$ is close to that of graphite. The radial Young's modulus of MWNTs has also been investigated by Palaci et al. ${ }^{17}$ and found to decrease with tube diameter, $D$, and reach to an asymptotic value, again close to the graphitic value. These results can be understood by the fact that the bulk modulus of these materials is essentially determined by a rather weak van der Waals $(\mathrm{vdW})$ interaction between tubes or between walls, which is similar to that between graphitic layers. Compared to these extensive studies on rather complicated but technologically attractive systems, the report on the radial elastic properties of isolated SWNTs is scarce. To our knowledge the first such report is from Reich et al. ${ }^{18}$ who performed ab initio calculations for a SWNT with $D=0.8 \mathrm{~nm}$ and obtained the radial Young's modulus of $E_{r} \approx 650 \mathrm{GPa}$, about half that in the axial direction. Recently, $\mathrm{Li}$ and $\mathrm{Chou}^{19}$ obtained somewhat larger $E_{r}$ using the molecular structural mechanics and found an inverse proportionality between $E_{r}$ and $D$, though approximate.

Radial deformation of carbon nanotubes has also attracted much attention because of its possible influence on the mechanical and electronic properties. Ruoff $e t$ al. ${ }^{20}$ observed, by transmission electron microscope, flattening of two adjacent aligned tubes along the contact region and showed that such a deformation can also be explained by a vdW interaction between tubes. Since then, the radial deformations of the carbon nanotubes in bundles and crystals have been investigated both experimentally and theoretically by many authors. ${ }^{15,16,21-29}$ These studies on bundles of SWNTs with particular diameters of $\sim 1.4 \mathrm{~nm}$ have suggested a pressureinduced phase transformation from circular to either hexagonal or flattened tubes at $1.4-2.1 \mathrm{GPa} .{ }^{15,21-25}$ This shape transition has been indirectly evidenced by the disappearance of symmetric radial breathing modes in Raman spectra. ${ }^{21,22}$ The shape transition has also been directly observed in the diffraction experiments, by either x-ray ${ }^{15,16}$ or neutron, ${ }^{27}$ although there remains inconsistency in these experimental results. The radial deformation of carbon nanotubes under asymmetric (or local) stress was also examined with molecular dynamics (MD) simulations $^{30}$ and scanning probe microscope.$^{31}$ In contrast with these extensive investigations for bundles and crystals, the radial deformation of isolated nanotubes has been much less studied. ${ }^{32-37}$ Chopra et al. ${ }^{33}$ observed, by transmission electron microscope, a completely 
collapsed (or flattened) MWNT and demonstrated, using a theoretical modeling, that such a collapsed tube is energetically favored over the inflated form for a given range of tube parameters. Sun et al. ${ }^{34}$ made more detailed investigations for the radial deformation of an isolated SWNT under pressure using classical and $a b$ initio constant-pressure MD simulations. They found that the cross section of a SWNT shows a circular-to-elliptical shape transition at some critical pressure, $P_{1}$, and the deformed elliptical phase is substantially soft compared to the circular phase with a large radial Young's modulus. The critical pressure was found to decrease with $D$ as $P_{1} \sim 1 / D^{3}$, which has also been suggested by other theoretical works ${ }^{19,25,34-36}$ and is in accordance with the classical continuum theory of buckling. Similar investigations for double-wall nanotubes were also made by Ye $e t$ $a l .{ }^{37}$ On the other hand, Zang et al. ${ }^{36}$ focused attention on a geometrical aspect of radial deformation of SWNTs and found, by a classical MD simulation, a universal feature related to the change in cross-sectional area.

In the present paper we are also concerned with the radial deformation and stability of isolated SWNTs under hydrostatic pressure. These results for a relatively simple system are expected to provide a useful understanding of the radial deformation in more complicated systems such as nanotube bundles, ropes, and crystals. The most promising theoretical method of studying deformation characteristics of carbon nanotubes would be to perform first-principles MD simulations, but this method requires prohibitively high cost of computations. To overcome this difficulty, we develop a theory enabling us to calculate the deformation energy of a SWNT much more easily. This theory starts with the results of $a b$ initio calculations based on the density functional theory (DFT) for the strain energy of an inflated SWNT with a circular cross section. We extend these results, using a continuum elastic shell model, to calculate the strain energy of a SWNT deformed in the radial direction without performing further $a b$ initio calculations. Then the theory is supplemented by adding the interaction between opposing walls, which is not fully taken into account or missing in the above modeling but becomes important in a highly deformed SWNT.

\section{METHOD TO CALCULATE DEFORMATION ENERGY}

Several atomistic, ${ }^{6,7,38}$ tight-binding, ${ }^{9}$ and $a b$ initio $^{39-44}$ calculations have been performed for the strain energy of inflated SWNTs with a circular cross section, which is the key quantity in the present theory. These calculations have indicated that the strain energy per atom is closely proportional to the inverse square of tube diameter, $D$, in a wide range of $D$ and insensitive to chirality of tube. We write this result, for later convenience, as

$$
E_{\mathrm{DFT}}=\frac{\alpha}{R^{2}}
$$

where $R$ is the tube radius $(R=D / 2)$ and $\alpha$ is a constant independent of $R$. The values of $\alpha$ obtained by the previous calculations are about $20 \mathrm{meV} \mathrm{nm}^{2}$. We also performed DFT calculations in the local density approximation (LDA) for
SWNTs with $D$ in the range $0.7-2.2 \mathrm{~nm}$, using the Vienna Ab Initio Simulation Package (VASP) code. ${ }^{46}$ In these calculations we assumed a SWNT directly constructed from a conformal mapping of graphene sheet with a C-C nearest neighbor distance of $0.142 \mathrm{~nm}$. To simulate an isolated SWNT we used a large supercell in the direction perpendicular to the axis. The calculations were made by using the ultrasoft pseudopotential and a plane-wave expansion with the cutoff energy of $358.2 \mathrm{eV}$. The $\mathbf{k}$-point sampling was made using special points in the irreducible Brillouin zone generated from $1 \times 1 \times 8$ mesh. By fitting the resulting strain energies to the form in Eq. (1), we obtained $\alpha=19.6$ $\mathrm{meV} \mathrm{nm}{ }^{2}$ for $(n, n)$ armchair tubes and $\alpha=19.2 \mathrm{meV} \mathrm{nm}^{2}$ for $(n, 0)$ zigzag tubes. In the theory developed in the following, we employ a continuum model in which carbon atoms are uniformly distributed on the conformal surface of a tube and hence no effect due to the chirality difference is considered. To be consistent with this, we used the average value, $\alpha$ $=19.4 \mathrm{meV} \mathrm{nm}^{2}$, throughout the present work. We note that no geometrical optimization was made in our calculations.

Kürti et $a l .{ }^{43}$ and Sun et al. ${ }^{45}$ have shown by the extensive DFT (LDA) calculations that the geometrical parameters of SWNTs differ from those obtained by the ideal wrapping of a graphene sheet. In fact, the unwrapped hexagonal lattice of a geometrically optimized SWNT is distorted and different from the ideal one, and the distortion of the unit cell (e.g., increase of the tube diameter) also occurs. These deviations from the ideal behaviors are appreciable for small SWNTs with $D \leqslant 1.5 \mathrm{~nm}$. The strain energy was also found to show the deviation from the classical behavior given by Eq. (1) and it may be corrected by adding extra terms such as that proportional to $R^{-4}$ if we are concerned with small tubes. ${ }^{41,43}$ In the present work, however, we ignore all these effects for avoiding complexity in treating them for deformed tubes in a consistent manner. In consequence, the present theory is quantitatively less reliable for small tubes, especially in the large deformation regime. For small deformations, with which we are primarily concerned in case of small tubes, the corrections due to the nonideal features of SWNTs can easily be estimated and will be found insignificant as we see later.

\section{A. Model}

Similar DFT calculations for a SWNT deformed in the radial direction would enable us to obtain its strain energy if we do not care about computational cost. Instead of attempting such unpractical calculations, we consider extending the result for a circular SWNT to a deformed one. For this purpose, we first note that the result in Eq. (1) is in accordance with that for a continuum elastic shell model, indicating that a SWNT may be viewed as a rolled-up elastic sheet with a thickness comparable to the interlayer distance of graphite. We also note the high stiffness in the axial direction, which suggests that the axial deformation induced by a radial deformation is quite small and may be ignored to a good approximation. The large circumferential Young's modulus ${ }^{19}$ also implies that the circumferential length of the cross section perpendicular to the axis remains almost unchanged in the course of radial deformation. Hence, we may assume that 
no deformation occurs in the axial direction and the circumferential length is always equal to $2 \pi R_{1}$, where $R_{1}$ is the radius when the deformation starts to occur. Let $\rho_{1}$ and $\alpha_{1}$ be the corresponding atomic number density on the surface and the value of $\alpha$ in Eq. (1), respectively. With these physically acceptable assumptions, the strain energy (per atom) of a deformed SWNT may be given by

$$
E_{\mathrm{DFT}}^{(0)}=\frac{1}{N} \oint \frac{\alpha_{1}}{R(s)^{2}} \rho_{1} L d s=\frac{\alpha_{1}}{2 \pi R_{1}} \oint \frac{1}{R(s)^{2}} d s .
$$

Here, $N=2 \pi R_{1} L \rho_{1}$ is the number of atoms in the length $L$ along the axis, $R(s)$ is the local curvature radius, and the integration is performed along the circumference in the plane perpendicular to the axis. The result in Eq. (2) is nothing but the one expected in the continuum elastic shell model and reduces to the form in Eq. (1) for a circular SWNT. As we see later, the radial deformation starts to occur at finite pressure, which implies that $R_{1}$ is smaller than the zero-pressure value, say $R_{0}$. The corresponding zero-pressure value of the density also would be smaller than $\rho_{1}$. However, these differences are insignificant and may be ignored for large tubes with $D \geqslant 1.5 \mathrm{~nm}$, as we see later. The situation is quite similar to that of the nonideal behaviors of the geometrical parameters and the strain energy. Hence, we may assume that $R_{1}, \alpha_{1}$, and $\rho_{1}$ are the same as the zero-pressure values, which we simply write as $R, \alpha$, and $\rho$ hereafter. The corrections due to these simplifications will be discussed later in Sec. III.

If the deformation is small and the interaction between opposing walls can be ignored, the continuum elastic sheet model as given by Eq. (2) may provide a good estimate of the strain energy of a deformed SWNT. However, in a situation where the deformation is large enough to cause the overlapping of the electron density in between opposing walls, the interaction between such walls becomes important. This interaction is similar to that between graphitic layers and certainly missing in the simple approximation given by Eq. (2). Such an extra contribution to the strain energy of a deformed SWNT may be taken into account by the method similar to that used to study interlayer interaction in graphite. ${ }^{47}$ By modifying that method, we may write the extra contribution as

$$
\begin{aligned}
E_{\mathrm{DFT}}^{(1)} & =\frac{1}{2 N} \int \rho d S \int \rho d S^{\prime} \phi_{\mathrm{DFT}}\left(\left|\mathbf{r}-\mathbf{r}^{\prime}\right|\right) G\left(\mathbf{r}, \mathbf{r}^{\prime}\right) \\
& =\frac{\rho^{2}}{2 N} \oint d s \oint d s^{\prime} \int_{-\infty}^{\infty} d z \int_{-L / 2}^{L / 2} d z^{\prime} \phi_{\mathrm{DFT}}\left(\left|\mathbf{r}-\mathbf{r}^{\prime}\right|\right) G\left(\mathbf{r}, \mathbf{r}^{\prime}\right) .
\end{aligned}
$$

Here, $N=2 \pi R L \rho$ as before, and $\phi_{\mathrm{DFT}}(r)$ is the interatomic interaction potential which is assumed to be that giving rise to the interlayer interaction of graphite obtained by the DFT calculations. ${ }^{47}$ In Eq. (3), the integrations in the first line over the conformal surface of a SWNT are written in the second line as those along the circumference and the $z$ axis taken to be in the axial direction, and $\mathbf{r}$ and $\mathbf{r}^{\prime}$ are the atomic positions on the circumferences specified by the variables $s$ and $s^{\prime}$, respectively. Finally, $G\left(\mathbf{r}, \mathbf{r}^{\prime}\right)$ is a geometrical factor

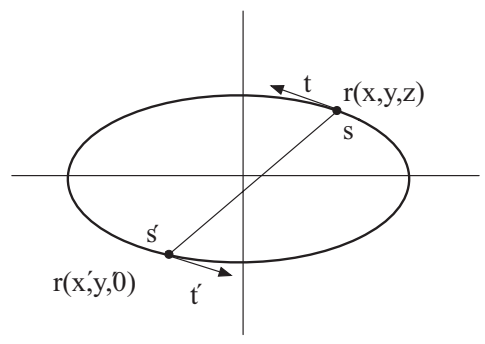

FIG. 1. Schematic view of the superimposed two circumferences of a cross section in the plane perpendicular to the tube axis. $\mathbf{t}$ and $\mathbf{t}^{\prime}$ are unit tangential vectors at the atomic positions on the two circumferences.

introduced to ensure that the atomic interaction already taken into account in Eq. (2) is excluded in Eq. (3), which implies the basic requirement that $G\left(\mathbf{r}, \mathbf{r}^{\prime}\right)=0$ for the two atoms on the same side of the wall and $G\left(\mathbf{r}, \mathbf{r}^{\prime}\right)=1$ for the two on the opposing walls. More detailed descriptions of $G\left(\mathbf{r}, \mathbf{r}^{\prime}\right)$ and its determination are given below. Before proceeding we note that by symmetry Eq. (3) reduces to

$$
E_{\mathrm{DFT}}^{(1)}=\frac{\rho}{2 \pi R} \oint d s \oint d s^{\prime} \int_{0}^{\infty} d z \phi_{\mathrm{DFT}}\left(\left|\mathbf{r}-\mathbf{r}^{\prime}\right|\right) G\left(\mathbf{r}, \mathbf{r}^{\prime}\right),
$$

with $\mathbf{r}=(x, y, z)$ and $\mathbf{r}^{\prime}=\left(x^{\prime}, y^{\prime}, 0\right)$. The geometrical situation related to Eqs. (3) and (4) is illustrated in Fig. 1.

One of the simplest forms of the geometrical factor satisfying the basic requirement may be given by

$$
G_{0}\left(\mathbf{r}, \mathbf{r}^{\prime}\right)=\frac{1}{2}\left(1-\mathbf{t} \cdot \mathbf{t}^{\prime}\right)
$$

where $\mathbf{t}$ and $\mathbf{t}^{\prime}$ are unit tangential vectors at $\mathbf{r}$ and $\mathbf{r}^{\prime}$, respectively, on the circumferences as shown in Fig. 1. If we use the above geometrical factor in Eq. (4), together with $\phi_{\mathrm{DFT}}(r)$ discussed later, we have non-negligible values of $E_{\mathrm{DFT}}^{(1)}$ for circular SWNTs. This result contradicts with another requirement that the extra contribution given by Eq. (4) should vanish for SWNTs with circular and slightly deformed cross section, for which the strain energy obtained by the DFT calculations is already taken into account in Eq. (1) or (2). This deficiency arises from that the interaction between atoms close to each other on the same side of the wall is improperly included in the calculations of $E_{\mathrm{DFT}}^{(1)}$ because $G_{0}\left(\mathbf{r}, \mathbf{r}^{\prime}\right)$ is not negligible for such a pair of atoms with $\mathbf{t} \cdot \mathbf{t}^{\prime} \approx 1$ (i.e., small $\theta$ with $\mathbf{t} \cdot \mathbf{t}^{\prime}=\cos \theta$ ) as shown in Fig. 2. We also note that interatomic interaction, $\phi_{\mathrm{DFT}}(r)$, obtained from the DFT calculations for graphite is that giving rise to the interaction between layers and cannot be used for the atoms on the same side of the wall. Also note that the extrapolated short-range part of $\phi_{\mathrm{DFT}}(r)$ is an artifact, as we will see later. The deficiency of $G_{0}\left(\mathbf{r}, \mathbf{r}^{\prime}\right)$ may be remedied by using an appropriate function with more radical behavior in the range where $\mathbf{t}$ and $\mathbf{t}^{\prime}$ are nearly parallel and antiparallel. After testing several such candidates, we found that one of the plausible functions is given by 


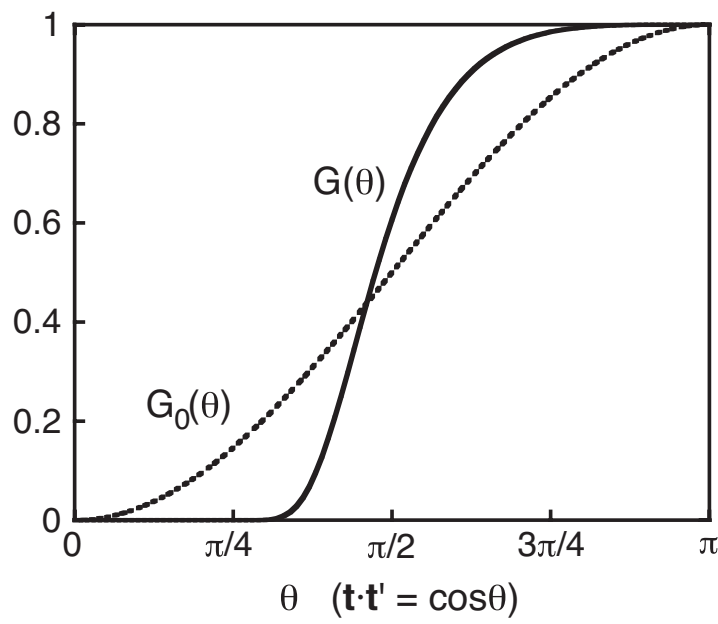

FIG. 2. Geometrical factors given by Eqs. (5) and (6). Here, $G_{0}\left(\mathbf{r}, \mathbf{r}^{\prime}\right)$ and $G\left(\mathbf{r}, \mathbf{r}^{\prime}\right)$ are written as $G_{0}(\theta)$ and $G(\theta)$, respectively, with $\mathbf{t} \cdot \mathbf{t}^{\prime}=\cos \theta$.

$$
\begin{aligned}
G\left(\mathbf{r}, \mathbf{r}^{\prime}\right) & =\exp \left[-\beta\left(\frac{1-G_{0}\left(\mathbf{r}, \mathbf{r}^{\prime}\right)}{G_{0}\left(\mathbf{r}, \mathbf{r}^{\prime}\right)}\right)^{m}\right] \\
& =\exp \left[-\beta\left(\frac{1+\mathbf{t} \cdot \mathbf{t}^{\prime}}{1-\mathbf{t} \cdot \mathbf{t}^{\prime}}\right)^{m}\right]
\end{aligned}
$$

This form is strictly ansatz but satisfies the basic requirement and shows the desired behavior with an appropriate choice of the parameters, $\beta$ and $m$. These parameters are determined so that the extra contribution given by Eq. (4) practically vanishes even for small SWNTs with $D$ as small as $0.8 \mathrm{~nm}$. However, only this requirement does not uniquely specify the parameters. In fact, if we use large values for both $\beta$ and $m$, we can always obtain $G\left(\mathbf{r}, \mathbf{r}^{\prime}\right)$ satisfying that condition. Hence, as a physically plausible condition, we required that we achieve $G(\mathbf{r}, \mathbf{r} \prime) \approx 1$ for nearly antiparallel $\mathbf{t}$ and $\mathbf{t}^{\prime}$ in the range as wide as possible, which implies that $\beta$ and $m$ are not too large. As a compromising set of values, we used $m=2$ and $\beta=0.5$ in the applications. The corresponding geometrical factor is also illustrated in Fig. 2. We have confirmed that the calculated strain energy is not sensitive to the choice of the parameters if they are not so far from the above choice.

Now the strain energy of a deformed SWNT is given by $E_{\mathrm{DFT}}=E_{\mathrm{DFT}}^{(0)}+E_{\mathrm{DFT}}^{(1)}$ and expected to be a good approximation to what we would have obtained by the full DFT calculations. Such DFT calculations in the local (LDA) and semilocal (GGA) approximations currently in use are quite successful in predicting bulk cohesive properties of solids but cannot capture the prevailing long-rage van der Waals (vdW) interaction, which is essentially the effect of the long-range electron correlation. The vdW interaction itself is not large and its contribution to the total cohesive energy of condensed phases is generally quite small (typically, less than 1\%) and this is the reason for the reasonable success of the conventional DFT calculations. However, the deficiency becomes serious when we are concerned with the properties of systems with sparse electron distribution. In fact, the contribution of the vdW interaction to the interlayer cohesive energy of graphite is substantial. ${ }^{47}$ This is also true for other graphitic systems such as fullerenes and carbon nanotubes. Hence, the above results for $E_{\mathrm{DFT}}$ based on the conventional DFT calculations are insufficient for our purpose of calculating the strain energy. Here we follow the common practice and assume that the vdW interaction between atoms is given by $^{48}$

$$
\phi_{\mathrm{vdW}}(r)=-\frac{A}{r^{6}} f_{\mathrm{damp}}(r)
$$

where $f_{\text {damp }}(r)$ is the damping function introduced for taking into account the fact that the asymptotic form, $-A / r^{6}$, obtained by the long-range multipole expansion is not valid at short distance and the potential should virtually vanish in the limit $r \rightarrow 0 .{ }^{48}$ In addition to this intrinsic requirement, we also note that the short-range part of the dispersion force interaction is already taken into account in the DFT calculations to some extent, depending on the DFT approximation. Hence, we may require that the damping function should also take account of that situation in addition to the intrinsic deficiency of the long-range multipole expansion. This implies that the damping function is no longer a universal function but depends on the DFT approximation used to calculate $E_{\mathrm{DFT}}$.

The vdW contribution to the total energy of a SWNT is then calculated in the way similar to that used to have Eq. (4) and given by

$$
E_{\mathrm{vdW}}=\frac{\rho}{2 \pi R} \oint d s \oint d s^{\prime} \int_{0}^{\infty} d z \phi_{\mathrm{vdW}}\left(\left|\mathbf{r}-\mathbf{r}^{\prime}\right|\right),
$$

with $\phi_{\mathrm{vdW}}(r)$ given by Eq. (7). We note that the geometrical factor is not involved in Eq. (8) because $E_{\mathrm{vdW}}$ is the contribution not included in the DFT calculations and the vdW interactions between all the pairs of atoms must be taken into account. Then, the total deformation energy defined with respect to a circular SWNT is given by

$$
\Delta E=\Delta E_{\mathrm{DFT}}^{(0)}+\Delta E_{\mathrm{DFT}}^{(1)}+\Delta E_{\mathrm{vdW}} .
$$

Here, $\Delta E_{\mathrm{DFT}}^{(0)}=E_{\mathrm{DFT}}^{(0)}-\alpha / R^{2}, \Delta E_{\mathrm{DFT}}^{(1)} \approx E_{\mathrm{DFT}}^{(1)}$ since $E_{\mathrm{DFT}}^{(1)}$ practically vanishes for a circular SWNT, and $\Delta E_{\mathrm{vdW}}=E_{\mathrm{vdW}}$ $-E_{\mathrm{vdW}}$ (circle), where $E_{\mathrm{vdW}}$ (circle) is the vdW contribution to the total energy of a circular SWNT.

\section{B. Determination of $\phi_{\mathrm{DFT}}(r)$ and $\phi_{\mathrm{vdW}}(r)$}

To determine the atomic interaction, $\phi_{\mathrm{DFT}}(r)$, in Eq. (4) we follow the previous work ${ }^{47}$ and assume that the interlayer interaction energy (per atom) of graphite obtained by the DFT calculations can be written as

$$
U_{\mathrm{DFT}}(d)=\frac{1}{2} \sum_{l=-\infty}^{\infty}{ }^{\prime} V_{\mathrm{DFT}}(l d)=\sum_{l=1}^{\infty} V_{\mathrm{DFT}}(l d),
$$

where $d$ is the interlayer distance, the prime on the summation implies the exclusion of the $l=0$ term, and $V_{\mathrm{DFT}}(l d)$ is the interaction energy between layers separated by $l d$. In the previous work, we found that $V_{\mathrm{DFT}}(l d)$ itself can be well represented by a Morse potential. ${ }^{47}$ Here, we take a different 


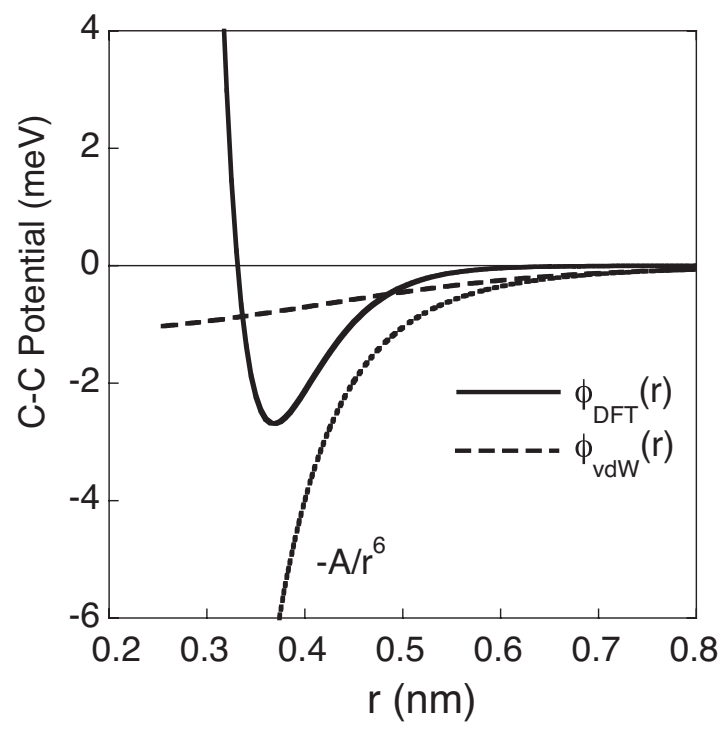

FIG. 3. Interatomic interactions, $\phi_{\mathrm{DFT}}(r)$ and $\phi_{\mathrm{vdW}}(r)$, obtained by the analysis of the interlayer cohesive energy of graphite.

approach and assume that $V_{\mathrm{DFT}}(l d)$ can be represented as the sum of the interatomic interactions between atoms on different layers, which is nothing but $\phi_{\mathrm{DFT}}(r)$ in Eqs. (3) and (4). Then, in the continuum model used throughout the present work, we have

$$
\begin{aligned}
V_{\mathrm{DFT}}(l d) & =\int_{0}^{\infty} \phi_{\mathrm{DFT}}\left(\sqrt{r^{2}+(l d)^{2}}\right) 2 \pi \rho r d r \\
& =2 \pi \rho \int_{l d}^{\infty} \phi_{\mathrm{DFT}}(r) r d r .
\end{aligned}
$$

We found that $\phi_{\mathrm{DFT}}(r)$ can also be well represented by a special type of the Morse potential,

$$
\phi_{\mathrm{DFT}}(r)=-M_{0}[1+\tau(x-1)] \exp [-\tau(x-1)],
$$

with $x=r / d_{\mathrm{M}}$. This form of the interatomic potential is nothing but the basis of the universal equation of state proposed by Vinet et al. ${ }^{49}$ The three parameters in $\phi_{\mathrm{DFT}}(r)$ were determined by fitting the resulting $U_{\mathrm{DFT}}(d)$ to the results of the previous DFT (LDA) calculations in the range, $d$ $=0.3-0.75 \mathrm{~nm} .{ }^{47}$ This DFT $(\mathrm{LDA})$ result for $U_{\mathrm{DFT}}(d)$ takes the minimum value of $-26.7 \mathrm{meV} /$ atom at $d=0.3318 \mathrm{~nm}$, which is very close to the experimental value of $d_{0}$ $=0.3337 \mathrm{~nm}$. The parameters yielding the optimum fit were found to be $M_{0}=2.689 \mathrm{meV}, \tau=9.902$, and $d_{\mathrm{M}}=0.3685 \mathrm{~nm}$. The performance of this fit is very good with the root-meansquare deviation less than $0.1 \mathrm{meV}$. The resulting $\phi_{\mathrm{DFT}}(r)$ is short range as illustrated in Fig. 3 and the interlayer interaction, $V_{\mathrm{DFT}}(l d)$, virtually vanishes for $l \geqslant 2$ even for $d$ as small as $0.3 \mathrm{~nm}$.

Similarly, the vdW contribution to the interlayer interaction is given by

$$
U_{\mathrm{vdW}}(d)=\sum_{l=1}^{\infty} V_{\mathrm{vdW}}(l d)
$$

with

$$
V_{\mathrm{vdW}}(l d)=2 \pi \rho \int_{l d}^{\infty} \phi_{\mathrm{vdW}}(r) r d r
$$

where $\phi_{\mathrm{vdW}}(r)$ is given by Eq. (7). The choice of the damping function is somewhat arbitrary and several forms have been proposed in the studies of intermolecular interaction. ${ }^{48}$ Here we employ the one-parameter form used by $\mathrm{Wu}$ and Yang, ${ }^{50}$

$$
f_{\text {damp }}(r)=\left\{1-\exp \left[-\left(r / R_{W}\right)^{3}\right]\right\}^{2} .
$$

If the damping function is practically unity beyond $r_{\max }$ $=l_{\max } d, U_{\mathrm{vdW}}(d)$ in Eq. (13) can be calculated as

$$
U_{\mathrm{vdW}}(d)=-\frac{\pi \rho A}{2 d^{4}}\left[\zeta(4)-4 \sum_{l=1}^{l_{\max }} J_{l}(d)\right],
$$

with

$$
J_{l}(d)=\int_{l}^{l_{\max }}\left[1-f_{\mathrm{damp}}(r)\right] x^{-5} d x \quad(x=r / d) .
$$

In Eq. (16), $\zeta(n)$ is the zeta function defined by $\zeta(n)=1$ $+1 / 2^{n}+1 / 3^{n}+\cdots$ and $\zeta(4)=\pi^{4} / 90$. The parameter in $f_{\text {damp }}(r)$ may be determined by requiring that the DFT result reinforced by the $\mathrm{vdW}$ contribution reproduces the interlayer cohesive energy, which is in the range $50-60 \mathrm{meV} /$ atom, as found by the recent theoretical and experimental analyses. ${ }^{47,51}$ In the present work we assumed

$$
U\left(d_{0}\right)=U_{\mathrm{DFT}}\left(d_{0}\right)+U_{\mathrm{vdW}}\left(d_{0}\right)=-55 \mathrm{meV} / \text { atom },
$$

as the compromising value of $U\left(d_{0}\right)$, where $d_{0}(=0.3337$ $\mathrm{nm})$ is the observed interlayer distance of graphite as before. Since $U_{\mathrm{DFT}}\left(d_{0}\right) \approx-27 \mathrm{meV} /$ atom, the $\mathrm{vdW}$ contribution to the interlayer cohesive energy of graphite is substantial. We used the value ${ }^{47} A=16.34 \times 10^{-6} \mathrm{eV} \mathrm{nm}^{6}$, as the vdW constant appropriate for carbon atoms in the $s p^{2}$ bonding state. ${ }^{50}$ Then, the requirement of Eq. (17) yields $R_{\mathrm{W}}=0.49 \mathrm{~nm}$, which implies that $l_{\max }=5$ at most. The resulting $\phi_{\mathrm{vdW}}(r)$ given by Eq. (7) is also shown in Fig. 3.

\section{Deformation model}

The radial deformation of SWNTs under hydrostatic pressure should be determined, at least in principle, by the condition that the stress tensor at each point on the tube surface balances the external force perpendicular to the surface. However, such a determination of the deformed shape is quite difficult in the present method based on the continuum model. To overcome this difficulty, we assumed plausible shapes of the cross section as illustrated in Fig. 4. In these model shapes the circumference of the cross section consists of smoothly connected four parts: two elliptic parts and two circular parts. The convex and concave types in Figs. 4(a) and 4(b), which we call respectively oval and peanut, are of 

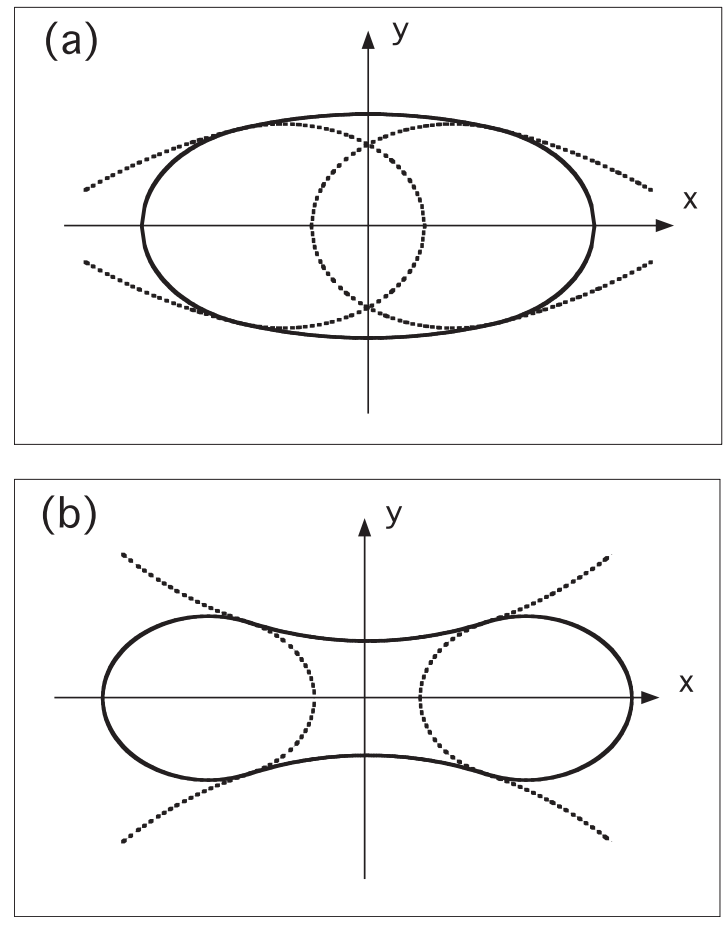

FIG. 4. Model shapes of the cross section of a deformed SWNT: (a) oval and (b) peanut. Both shapes reduce to an ellipse as a whole when the centers of two ellipses on both sides coincide with each other. Each shape, either oval or peanut, transforms into another through a racetracklike shape, which corresponds to the infinite radius of two circles on the top and bottom.

identical shape in a special case where the centers of two ellipses on both sides coincide with each other. In such a case both shapes reduce to an ellipse as a whole. Also note that each type in Fig. 4 is transformed into another through the flattened racetracklike shape corresponding to the infinite curvature radius of circular parts on the top and bottom. The model cross sections in Fig. 4 are characterized by four parameters once we have imposed the condition that four parts are smoothly connected. One of these parameters can be eliminated by the assumption that the length of the conformal circumference remains unchanged, which we discussed earlier. Then, for a given fractional change (decrease) in volume,

$$
X=1-V / V_{0}=1-S / S_{0} \quad\left(S_{0}=\pi R^{2}\right),
$$

the two remaining parameters are determined so as to minimize the deformation energy given by Eq. (9). In Eq. (18), $S$ is the cross-sectional area of a deformed SWNT and we used, as before, the assumption that no deformation occurs in the axial direction. We may expect that the deformation optimized in this way for a given $X$ is, though approximately, consistent with the applied hydrostatic pressure. In fact, the model cross-sectional shapes in Fig. 4 are quite similar to those predicted by constant-pressure molecular dynamics (MD) simulations using the empirical potentials for atomic interaction. ${ }^{34-37}$

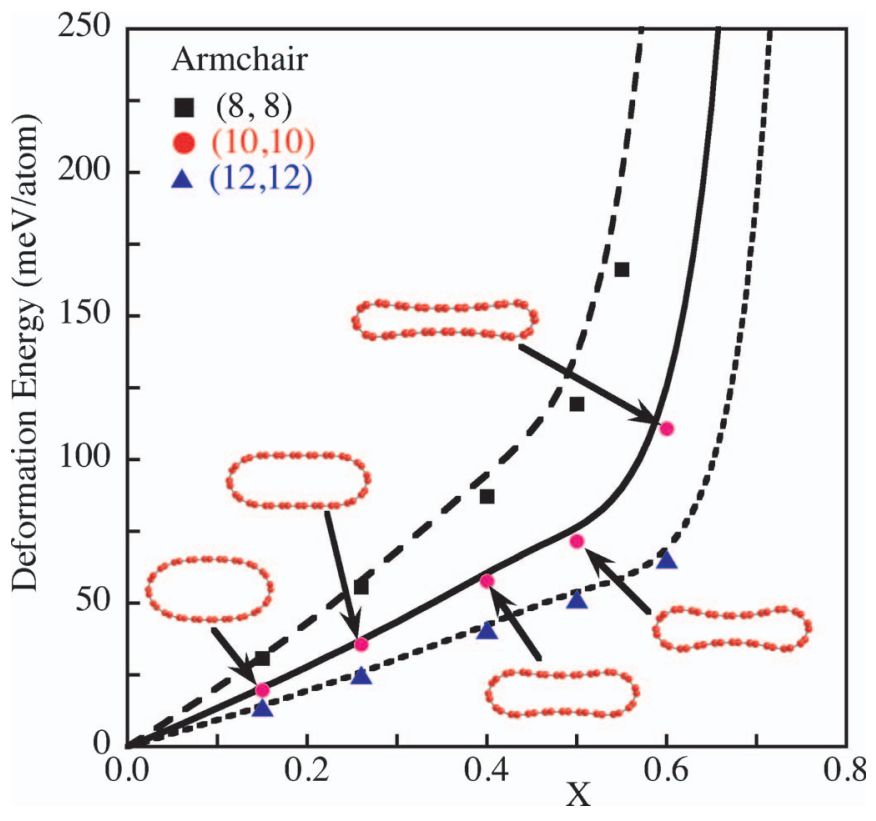

FIG. 5. (Color online) Deformation energies as functions of the fractional volume decrease, $X$, defined by Eq. (18). The full DFT (LDA) results for the armchair $(8,8),(10,10)$, and $(12,12)$ SWNTs (squares, circles, and triangles, respectively) are compared with the DFT contributions, $\Delta E_{\mathrm{DFT}}=\Delta E_{\mathrm{DFT}}^{(0)}+\Delta E_{\mathrm{DFT}}^{(1)}$, obtained by the present continuum model for the corresponding tubes with $D=1.085 \mathrm{~nm}$ (broken line), $D=1.356 \mathrm{~nm}$ (solid line), and $D=1.627 \mathrm{~nm}$ (dotted line). The cross sections of the unit sells used in the full DFT (LDA) calculations are also shown for the deformed $(10,10)$ tubes.

\section{RESULTS OF APPLICATION}

Before proceeding to the systematic applications, we tested the present theory for particular SWNTs with relatively small diameters. In this test, we first calculated the deformation energies by using the method described in the previous section. For the deformed SWNTs with the crosssectional shapes determined in this way, we also performed full DFT (LDA) calculations. The carbon atoms are now arranged by the ideal mapping on that deformed tube surface. Figures 5 and 6 show the comparisons of these DFT (LDA) results with the corresponding contributions in Eq. (9), $\Delta E_{\mathrm{DFT}}=\Delta E_{\mathrm{DFT}}^{(0)}+\Delta E_{\mathrm{DFT}}^{(1)}$, obtained by the present model. For the armchair $(12,12)$ tube $(D=1.627 \mathrm{~nm})$, we find a reasonable agreement between the full DFT and model calculations even for a highly deformed tube (Fig. 5). The agreement is less favorable for smaller tubes as exemplified for the (10, $10)$ and $(8,8)$ tubes with $D=1.356 \mathrm{~nm}$ and $1.085 \mathrm{~nm}$, respectively. Better agreement is achieved for the zigzag tubes as we see in Fig. 6 for the $(18,0)$ tube with the diameter $(D$ $=1.409 \mathrm{~nm})$ comparable to that of $(10,10)$ tube, suggesting that the present model better describes the deformation of zigzag tubes. For the $(12,0)$ tube $(D=0.940 \mathrm{~nm})$, we find a somewhat large deviation in the large deformation regime. All these results suggest the validity as well as usefulness of the present theory for calculating the deformation energies of relatively large SWNTs with $D \geqslant 1.5 \mathrm{~nm}$.

Figure 6 also shows how the calculated deformation energies of the (18.0) tube change as we successively evolve 


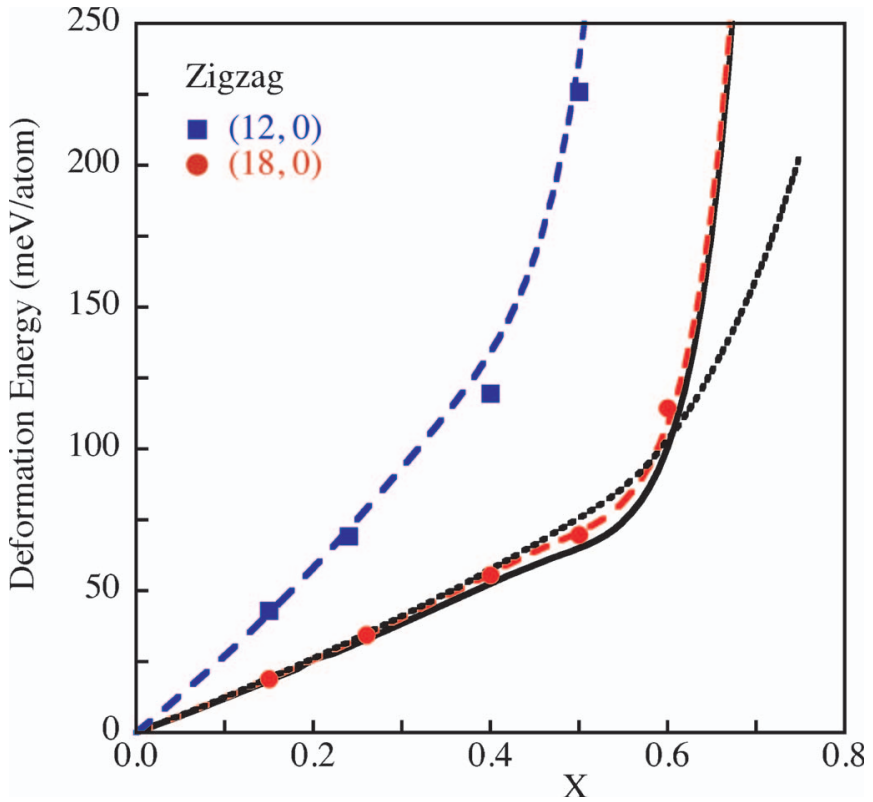

FIG. 6. (Color online) Deformation energies of the zigzag (12, $0)$ and $(18,0)$ SWNTs obtained by the full DFT (LDA) calculations (squares and circles, respectively) and their comparisons with the DFT contributions (broken lines), $\Delta E_{\mathrm{DFT}}=\Delta E_{\mathrm{DFT}}^{(0)}+\Delta E_{\mathrm{DFT}}^{(1)}$, obtained by the present continuum model for the corresponding tubes with $D=0.940 \mathrm{~nm}$ and $D=1.409 \mathrm{~nm}$. For the latter tube $(D=1.409 \mathrm{~nm})$, the deformation energies with the successive inclusions of the contributions interwall and vdW interactions are also shown: $\Delta E_{\mathrm{DFT}}^{(0)}$ (dotted line), $\Delta E_{\mathrm{DFT}}$ (broken line), and $\Delta E$ given by Eq. (9) (solid line). The cross-sectional shapes of the deformed $(18,0)$ tube are similar to those of the $(10,10)$ tube (Fig. 5).

the model by including the contributions due to the interaction between opposing walls and the vdW interaction. In the small deformation regime, $X \leqslant 0.4$, the extended classical result given by Eq. (2) yields quite a good estimate of the deformation energy. The contribution due to the interwall interaction is appreciable for $X>0.4$ and becomes comparable to that due to the vdW interaction at $X \approx 0.5$, where the smallest distance between the opposing walls is close to the interlayer distance of graphite. This result is quite natural since the flattened SWNT is similar to the system consisting of two parallel graphene sheets. As the tube deforms further, the distance between the flattened walls decreases and the interaction between them becomes repulsive (Fig. 3), which causes the rapid increase of $\Delta E_{\mathrm{DFT}}$ for $X>0.5$. For smaller
SWNTs, especially in the large deformation regime, the present model calculations are less accurate and have a room for improvement, as we discussed earlier. In fact, the smallest local curvature radius of the highly deformed $(10,10)$ tube at $X=0.6$ (Fig. 5) is about $0.22 \mathrm{~nm}(0.44 \mathrm{~nm}$ in diameter), suggesting that the ideal behavior of the strain energy given by Eq. (1) needs a modification. However, we also note that such a large deformation occurs only at an extremely high pressure, as we will see later, and at this stage such an extreme case is outside our interest.

We now examine possible influences of the nonideal behaviors of the geometrical parameters ${ }^{43,45}$ on the deformation energy of small SWNTs. As a typical example of such tubes, we consider the armchair $(6,6)$ SWNT with the ideal diameter of $0.8136 \mathrm{~nm}$. The optimized diameter of this tube is $\sim 0.820 \mathrm{~nm}$, about $0.75 \%$ larger than the ideal value, and the strain energy deviate upwards by $\sim 1 \%$ from the ideal result given by Eq. (1). ${ }^{43}$ The former has the effect of lowering the strain energy by about $1.5 \%$ in the small deformation regime [see Eq. (2)] and, in total, the deformation energy of the actual $(6,6)$ tube should be lower by $\sim 0.5 \%$ than that obtained for the ideal tube. If we use the optimized diameter $(0.820 \mathrm{~nm})$ rather than the ideal value $(0.8136 \mathrm{~nm})$ for this tube, we underestimate the deformation energy by $\sim 1 \%$ in the small deformation regime by using the uncorrected result given by Eq. (1). In this way we can estimate the corrections due to the nonideal behaviors of the geometrical parameters and the strain energy. These corrections are within a few percent for tubes as small as the $(6,6)$ SWNT. As we have pointed out earlier and will see later, the radial deformation of a tube starts to occur at finite pressure, $P_{1}$. Sun et al. ${ }^{34}$ performed $a b$ initio molecular dynamics simulations for the $(6,6)$ tube and obtained $P_{1} \approx 10 \mathrm{GPa}$. On the other hand, Reich et al. ${ }^{18}$ predicted the radial bulk modulus for this tube as $E_{r} \approx 650 \mathrm{GPa}$. These results imply that the tube uniformly shrinks by about $1.5 \%$ under the transition pressure. Hence, taking into account $\sim 0.75 \%$ increase due to nonideal behavior, ${ }^{43}$ we find that the diameter of this tube will be smaller by $\sim 0.75 \%$ than the ideal value and the correction to the deformation energy due to this effect is about $1.5 \%$. The finite $P_{1}$ effect is the smaller for the larger tube because $E_{r}$ and $P_{1}$ show the behaviors $E_{r} \propto 1 / D$ as predicted by Reich $e t$ al. ${ }^{18}$ and $P_{1} \propto 1 / D^{3}$ as we see later.

We now proceed to the results of the systematic applications of the present theory to SWNTs with wide range of diameters and show the calculated deformation energies as functions of $X$ in Fig. 7. We first note that the value of $X$ at
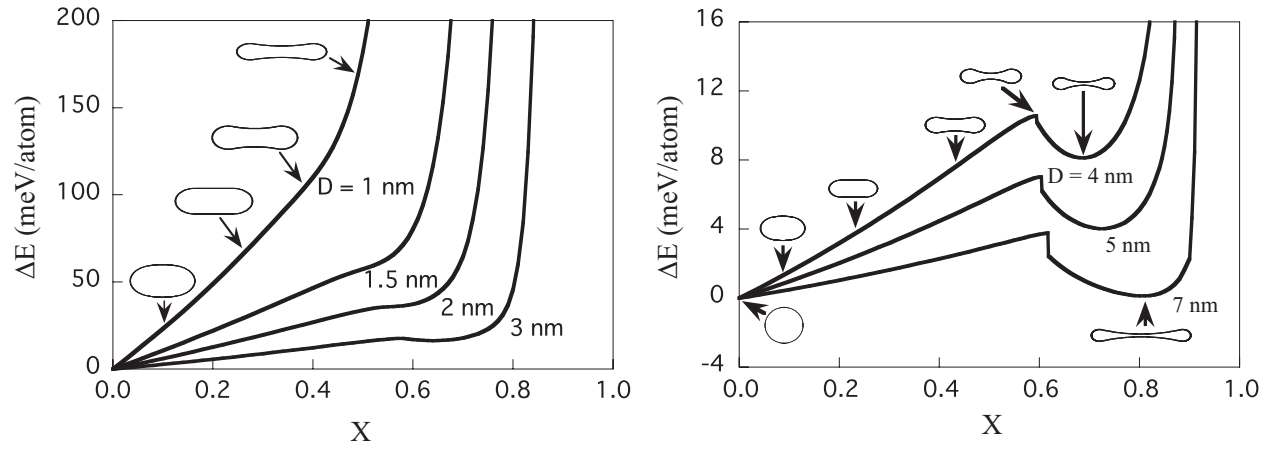

FIG. 7. Deformation energies obtained by the present method for SWNTs with $D=1-7 \mathrm{~nm}$ as functions of $X$. The optimized shapes at selected values of $X$ are also shown. 
which the oval-to-peanut transition occurs is 0.22 for the tube with $D=0.8 \mathrm{~nm}$, increases with $D$ up to $D \approx 1 \mathrm{~nm}$, and remains unchanged at $X \approx 0.26$ for larger tubes with $D$ $>1 \mathrm{~nm}$. At these values of $X$, which we denote $X_{2}$, the crosssectional shape is racetracklike. In the small deformation regime, say $X<X_{c}$, the interaction between the opposing walls hardly contributes to the deformation energy, $\Delta E$. The value of $X_{c}$ depends on the size of SWNT and lies in the range from 0.40 (for $D=1 \mathrm{~nm}$ ) to 0.53 (for $D=8 \mathrm{~nm}$ ). In that regime, $\Delta E$ is essentially given by that of the continuum elastic shell model and the contribution, $\Delta E_{\mathrm{DFT}}^{(0)}$, in Eq. (9) dominates the deformation energy as we have seen in Fig. 6 for a particular case. In consequence, $\Delta E$ nearly scales as $R^{-2}$, implying that the deformation in that regime has a universal feature. In fact, the constancy of $X_{2}$ for tubes with $D$ $>1 \mathrm{~nm}$ may be explained by that feature of the deformation. Zang et al. ${ }^{36}$ have also investigated this universal feature of the shape transition using a constant-pressure MD method. They demonstrated that the ratio of the cross-sectional areas at the two shape transitions is independent of diameter and chirality of SWNTs and given by $S_{2} / S_{1} \approx 0.82$ which corresponds to $X_{2}=0.18$, where $S_{1}$ is the cross-sectional area at the first transition from circle to oval $\left(S_{1}=S_{0}=\pi R^{2}\right.$ in the present case) and $S_{2}$ is the one at the second transition from oval to peanut. The present result for the second transition, $X_{2}$ $=0.22-0.26$, is somewhat larger than that predicted by Zang et al. ${ }^{36}$ and the difference may be ascribed to our choice of model cross-sectional shape. However, we also note that the variation of $\Delta E$ with shape change about the second transition is very small, implying that it is quite difficult to pinpoint that transition in simulations.

The optimized cross-sectional shapes are peanuts for large deformations beyond $X=X_{2}$, but the deformation pattern changes at $X=X_{c}$, where the attractive interaction between the concave parts of wall becomes appreciable and the deformation energy tends to be flat as a function of $X$ beyond $X=X_{c}$ as we have already seen in Fig. 6. At the first stage, $X<X_{c}$, the shape of peanut becomes more concave. On the other hand, the deformation in the range $X>X_{c}$ is characterized by stretch rather than concaving. This feature may be explained by the fact that stretch gives rise to the increase in area of opposing flattened walls and is energetically favorable owing to the contribution of $\Delta E_{\mathrm{DFT}}^{(1)}$ and $\Delta E_{\mathrm{vdW}}$ in Eq. (9). If the cross section is deformed further beyond $X=X_{c}$, the deformation energy, $\Delta E$, of large SWNTs with $D$ $\geqslant 2.5 \mathrm{~nm}$ starts to decrease at $X=X_{\text {collapse, }}$ i.e., the deformation proceeds spontaneously and the tube collapses. This decrease in $\Delta E$ is discontinuous for large SWNTs with $D$ $\geqslant 4 \mathrm{~nm}$ and the discontinuity of $\Delta E$ increases with tube diameter. The minimum of $\Delta E$ is achieved at $X=0.7-0.9$ for the tubes with $D=4-8 \mathrm{~nm}$ and at this deformation the smallest distance between the flattened walls is about $0.3 \mathrm{~nm}$, which is comparable to the interlayer spacing of graphite. If a SWNT is further deformed the distance between such walls decreases and the repulsive interaction between them through $\phi_{\mathrm{DFT}}(r)$ becomes substantial. The sharp rise of $\Delta E$ with increasing $X$ beyond the minimum point is mostly owing to this effect. We also note that the minimum value of $\Delta E$ becomes negative for large SWNTs with $D>7 \mathrm{~nm}$, i.e., the collapsed state is favored over the inflated one with circular cross section. This critical size of $D \approx 7 \mathrm{~nm}$ is somewhat larger than that predicted by Chopra et al. ${ }^{33} D \approx 5.3 \mathrm{~nm}$. The difference between the present results and those of Chopra et al. may be explained mostly by their simplified treatment of deformation. They assumed a dumbbell-type shape with flat and parallel walls, but such a shape has not been observed in any constant-pressure simulation for an isolated SWNT and certainly gives an overestimation of the attractive interaction between the flattened walls.

In contrast to these characteristics for large tubes with $D \geqslant 2.5 \mathrm{~nm}$, the deformation energy of small tubes with $D$ $<2.5 \mathrm{~nm}$ monotonically increases with $X$ and the tubes show no tendency to collapse up to very large deformation. This behavior of $\Delta E$ may be ascribed to the energy gain due to the attractive interaction, which is minimal for small tubes and, if any, it is compensated by the energy loss due to either the curvature effect on both sides of the stretched peanut or the repulsive interaction between walls, which become appreciable at a relatively small deformation, as we have seen in Fig. 6. These results for $\Delta E$ indicate that even if a tube is highly deformed under pressure, it returns to the normal shape with circular cross section on pressure release, i.e., the deformation is reversible (elastic). These deformation characteristics found for isolated SWNTs with small diameter may explain the experimental fact that the radial deformation of nanotube bundles is elastic up to very high pressure. ${ }^{16}$

As we have already discussed, we assumed that our model deformation of an isolated SWNT is consistent with the external force due to the hydrostatic pressure. Then, the balance between the work done by pressure, $P$, and the increase in the deformation energy due to the radial deformation implies the relation

$$
P d V=-2 \pi R \rho\left(\frac{\partial \Delta E}{\partial V}\right) d V=\frac{2 \rho}{R}\left(\frac{\partial \Delta E}{\partial X}\right) d V .
$$

Then, the minimum pressure for the deformation to occur, i.e., the circular-to-oval transition pressure, is given by

$$
P_{1}=\frac{2 \rho}{R}\left(\frac{\partial \Delta E}{\partial X}\right) \quad(X=0) .
$$

This result indicates that $P_{1}$ is closely proportional to the inverse cube of the tube radius since the deformation energy, $\Delta E$, itself scales as $R^{-2}$ in the small deformation regime, where the contribution $\Delta E_{\mathrm{DFT}}^{(0)}$ in Eq. (9) dominates $\Delta E$, as we discussed earlier. In fact, the calculated values of $P_{1}$ fit quite well to the form, $\gamma / R^{3}$, with $\gamma \approx 5.5 \mathrm{GPa} \mathrm{nm}^{3}$, as shown in Fig. 8. This result is in agreement with that suggested by other theoretical works, ${ }^{19,25,34-36}$ as we have noted earlier. The pressure given by Eq. (20) is also nothing but the hard-to-soft transition pressure studied by Sun et al. ${ }^{34}$ The present result for the armchair $(6,6)$ SWNT $(D=0.8136$ $\mathrm{nm}$ in the present model $), P_{1} \approx 9.70 \mathrm{GPa}\left(P_{1} \approx 9.95 \mathrm{GPa}\right.$ if corrected as we have discussed earlier), is in good agreement with their result, $P_{1} \approx 10 \mathrm{GPa}$, proving the validity as well as the usefulness of the present method based on the continuum elastic shell model. The oval-to-peanut transition pressure is given by 


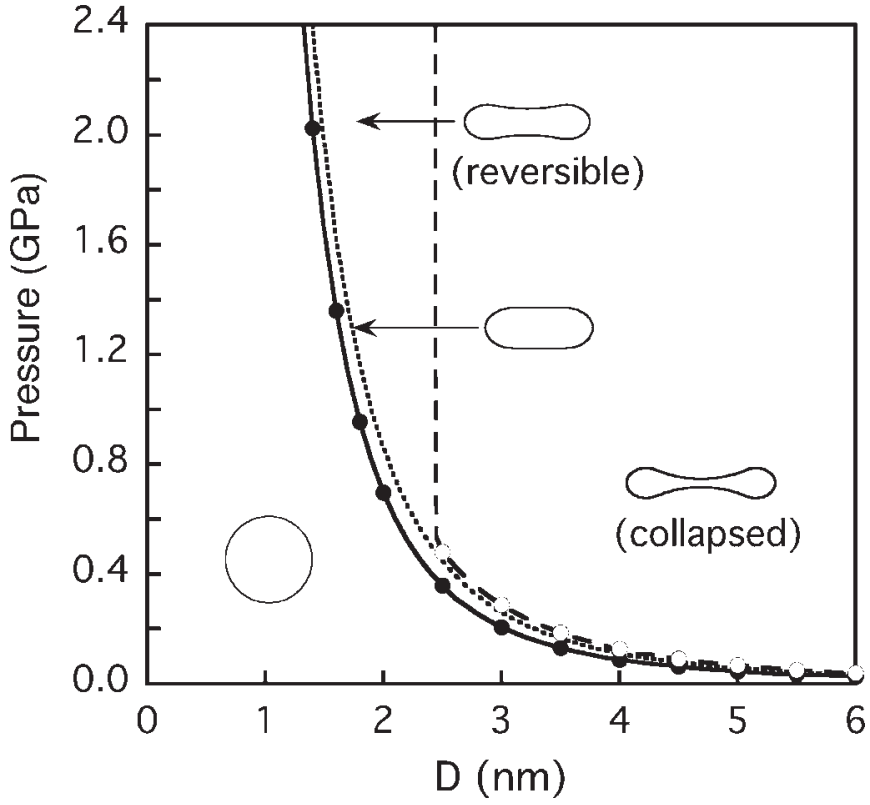

FIG. 8. Circular-to-oval transition pressure $\left(P_{1}\right.$, closed circles), oval-to-peanut transition pressure $\left(P_{2}\right.$, broken line), and collapse pressure $\left(P_{\text {collapse }}\right.$, open circles) as functions of tube diameter. The solid and broken lines are smooth interpolations of the calculated results.

$$
P_{2}=\frac{2 \rho}{R}\left(\frac{\partial \Delta E}{\partial X}\right) \quad\left(X=X_{2}\right)
$$

where $X_{2}$ is, as before, the value of $X$ at which the optimum cross-sectional shape is racetracklike. The pressure given by Eq. (21) is also shown in Fig. 6 and found to be $20-30 \%$ higher than $P_{1}$.

For the particular SWNTs with $D \approx 1.4 \mathrm{~nm}$, we find that the circular-to-oval transition occurs at $P_{1} \approx 2.0 \mathrm{GPa}$. This transition pressure is close to the experimental results for bundles of SWNT with similar $D, 1.5-1.7 \mathrm{GPa}$, at which a shape transition was indirectly evidenced by Raman spectroscopy. ${ }^{21,22}$ Using MD simulations for bundles of (10, 10) nanotubes $(D=1.36 \mathrm{~nm})$, Elliott et al. ${ }^{25}$ found that these nanotubes transform from circular to herringbone structure at $2.1 \pm 0.2 \mathrm{GPa}$. This pressure is also close to the present result, $P_{1} \approx 2.2 \mathrm{GPa}$, for the corresponding isolated tube. However, we should note that the interaction between tubes could have some effect on the transition and the results for bundles might not directly be compared with those for isolated tubes. Under high pressure beyond $P_{2}$, the cross section of isolated SWNTs certainly takes a shape of peanut as predicted by the present calculations and the previous MD simulations. ${ }^{34,36}$ In contrast, the flattened shape similar to a racetrack is favored in bundles $24,25,29$ This result for bundles may be explained by the contribution due to the attractive interaction between tubes, which is large in the arrangement where the surfaces of neighboring tubes are flat and parallel to each other.

As we have discussed, SWNTs with large diameters $(D$ $\geqslant 2.5 \mathrm{~nm}$ ) collapse if they are deformed down to the critical volume characterized by $X_{\text {collapse }}$, and the collapse pressure is given by

$$
P_{\text {collapse }}=\frac{2 \rho}{R}\left(\frac{\partial \Delta E}{\partial X}\right)_{\max },
$$

where $(\partial \Delta E / \partial X)_{\max }$ is the maximum slope of the $\Delta E$ versus $X$ curve in the range, $0<X<X_{\text {collapse }}$. The slope is the maximum at $X \approx 0.6$ independent of tube size and $P_{\text {collapse }}$ is about 40-50\% larger than $P_{1}$, as illustrated in Fig. 8. At pressures lower than $P_{\text {collapse }}$, tubes are not deformed to the critical volume and inflate on pressure release, i.e., the deformation is elastic in the pressure range between $P_{1}$ and $P_{\text {collapse }}$. On the other hand, the $\Delta E$ versus $X$ curves for small SWNTs with $D \leqslant 2.5 \mathrm{~nm}$ have no local minimum [Fig. 7(a)], indicating that the collapse due to radial deformation does not occur and, if they actually do collapse, it should be ascribed to other causes such as the breaking of $\mathrm{C}-\mathrm{C}$ bond or defect formation.

\section{CONCLUSIONS}

We have developed a simple theory for calculating the deformation energies of SWNTs and applied it to study the radial deformation and stability. The first step of the theory is to extend the result of DFT calculations for the strain energy of circular SWNTs to a deformed SWNT using the continuum elastic shell model in the linear elastic regime. Then this model was supplemented by the interactions between opposing walls, which become important for highly deformed tubes and plays a crucial role in determining the deformation characteristics, including collapse. One of these interactions is that we could have taken into account in a full DFT calculation for a deformed tube but certainly miss in the simple extension based on the continuum elastic shell model as given by Eq. (2). The other one is the vdW interaction, which the DFT approximations currently in use fail to take into account. Both interactions are deduced from the semiempirical analyses of the interlayer cohesive energy of graphite. $^{47}$

The theory provides a simple method to calculate deformation energy of isolated SWNTs under hydrostatic pressure. The shape transitions predicted for SWNTs with particular diameters are in accordance with the results of the existing MD simulations, $a b$ initio calculations, and experiments, suggesting the validity and usefulness of the present theory. The energetics of collapse due to the radial deformation were revealed. The theory may also be applied to other systems such as MWNTs and bundles of nanotubes. However, the most serious difficulty in such applications is that we need to devise a parameterized model for the crosssectional shape of the tube.

\section{ACKNOWLEDGMENTS}

We thank T. Sasaki for useful discussion. This work was supported in part by a Grant-in-Aid for Scientific Research from the JSPS under Grants No. 16540301 and No. 16550002. The computations in the present work were performed using the facilities of the Information Processing Center of Iwate University and the Supercomputer Center of the Institute for Solid State Physics, University of Tokyo. 
*Electronic address: hasegawa@iwate-u.ac.jp

${ }^{1}$ S. Iijima, Nature (London) 354, 56 (1991).

${ }^{2}$ R. Saito, G. Dresselhaus, and M. S. Dresselhaus, Physical Properties of Carbon Nanotubes (Imperial College Press, London, 1998).

${ }^{3}$ M. M. J. Treacy, T. W. Ebbersen, and J. M. Gibson, Nature (London) 381, 678 (1996).

${ }^{4}$ E. W. Wong, P. E. Sheehan, and C. M. Lieber, Science 277, 1971 (1997).

${ }^{5}$ J.-P. Salvetat, G. A. Briggs, J.-M. Bonard, R. R. Bacsa, A. J. Kulik, T. Stockli, N. A. Burnham, and L. Forro, Phys. Rev. Lett. 82, 944 (1999).

${ }^{6}$ D. H. Robertson, D. W. Brenner, and J. W. Mintmire, Phys. Rev. B 45, 12592 (1992).

${ }^{7}$ B. I. Yakobson, C. J. Brabec, and J. Bernholc, Phys. Rev. Lett. 76, 2511 (1996).

${ }^{8}$ J. P. Lu, Phys. Rev. Lett. 79, 1297 (1997).

${ }^{9}$ E. Hernandéz, C. Goze, P. Bernier, and A. Rubio, Phys. Rev. Lett. 80, 4502 (1998).

${ }^{10}$ A. Sears and R. C. Batra, Phys. Rev. B 69, 235406 (2004).

${ }^{11}$ T. Vodenitcharova and L. C. Zhang, Phys. Rev. B 68, 165401 (2003).

${ }^{12}$ L. Wang, Q. Zheng, J. Z. Liu, and Q. Jiang, Phys. Rev. Lett. 95, 105501 (2005).

${ }^{13}$ J. Tersoff and R. S. Ruoff, Phys. Rev. Lett. 73, 676 (1994).

${ }^{14}$ V. N. Popov, V. E. V. Doren, and M. Balkanski, Solid State Commun. 114, 395 (2000).

${ }^{15}$ J. Tang, L.-C. Qin, T. Sasaki, M. Yudasaka, A. Matsushita, and S. Iijima, Phys. Rev. Lett. 85, 1887 (2000).

${ }^{16}$ S. M. Sharma, S. Karmakar, S. K. Sikka, P. V. Teredesai, A. K. Sood, A. Govindaraj, and C. N. R. Rao, Phys. Rev. B 63, 205417 (2001).

${ }^{17}$ I. Palaci, S. Fedrigo, H. Brune, C. Klinke, M. Chen, and E. Riedo, Phys. Rev. Lett. 94, 175502 (2005).

${ }^{18}$ S. Reich, C. Thomsen, and P. Ordejón, Phys. Rev. B 65, 153407 (2002).

${ }^{19}$ C. Li and T.-W. Chou, Phys. Rev. B 69, 073401 (2004).

${ }^{20}$ R. S. Ruoff, J. Tersoff, D. C. Lorents, S. Subramoney, and B. Chan, Nature (London) 364, 514 (1993).

${ }^{21}$ U. D. Venkateswaran, A. M. Rao, E. Richter, M. Menon, A. Rinzler, R. E. Smalley, and P. C. Eklund, Phys. Rev. B 59, 10928 (1999).

${ }^{22}$ M. J. Peters, L. E. McNeil, J. P. Lu, and D. Kahn, Phys. Rev. B 61, 5939 (2000).

${ }^{23}$ M. J. López, A. Rubio, J. A. Alonso, L.-C. Qin, and S. Iijima, Phys. Rev. Lett. 86, 3056 (2001).

${ }^{24}$ S.-P. Chan, W.-L. Yim, X. G. Gong, and Z.-F. Liu, Phys. Rev. B 68, 075404 (2003).

${ }^{25}$ J. A. Elliott, J. K. W. Sandler, A. H. Windle, R. J. Young, and M.
S. P. Shaffer, Phys. Rev. Lett. 92, 095501 (2004); J. A. Elliott, J. K. W. Sandler, A. H. Windle, R. J. Young, and M. S. P. Shaffer, ibid. 93, 149602 (2004).

${ }^{26}$ S. A. Chesnokov, V. A. Nalimova, A. G. Rinzler, R. E. Smalley, and J. E. Fischer, Phys. Rev. Lett. 82, 343 (1999).

${ }^{27}$ S. Rols, I. N. Gontcharenko, R. Almairac, J. L. Sauvajol, and I. Mirebeau, Phys. Rev. B 64, 153401 (2001).

${ }^{28}$ M. H. F. Sluiter, V. Kumar, and Y. Kawazoe, Phys. Rev. B 65, 161402(R) (2002).

${ }^{29}$ X. H. Zhang, D. Y. Sun, Z. F. Liu, and X. G. Gong, Phys. Rev. B 70, 035422 (2004).

${ }^{30}$ V. Gadagkar, P. K. Maiti, Y. Lansac, A. Jagota, and A. K. Sood, Phys. Rev. B 73, 085402 (2006).

${ }^{31}$ V. Lordi and N. Yao, J. Chem. Phys. 109, 2509 (1998).

${ }^{32}$ W. Shen, B. Jiang, B. S. Han, and S. S. Xie, Phys. Rev. Lett. 84, 3634 (2000).

${ }^{33}$ N. G. Chopra, L. X. Benedict, V. H. Crespi, M. L. Cohen, S. G. Louie, and A. Zettle, Nature (London) 377, 135 (1995).

${ }^{34}$ D. Y. Sun, D. J. Shu, M. Ji, F. Liu, M. Wang, and X. G. Gong, Phys. Rev. B 70, 165417 (2004).

${ }^{35}$ R. B. Capaz, C. D. Spataru, P. Tangney, M. L. Cohen, and S. G. Louie, Phys. Status Solidi B 241, 3352 (2004).

${ }^{36}$ J. Zang, A. Treibergs, Y. Han, and F. Liu, Phys. Rev. Lett. 92, 105501 (2004)

${ }^{37}$ X. Ye, D. Y. Sun, and X. G. Gong, Phys. Rev. B 72, 035454 (2005).

${ }^{38}$ L. Shen and J. Li, Phys. Rev. B 71, 165427 (2005).

${ }^{39}$ J. W. Mintmire and C. T. White, Carbon 33, 893 (1995).

${ }^{40}$ D. Sánchez-Portal, E. Artacho, J. M. Soler, A. Rubio, and P. Ordejon, Phys. Rev. B 59, 12678 (1999).

${ }^{41}$ K. Kanamitsu and S. Saito, J. Phys. Soc. Jpn. 71, 483 (2002).

${ }^{42}$ O. Gülseren, T. Yildirim, and S. Ciraci, Phys. Rev. B 65, 153405 (2002).

${ }^{43}$ J. Kürti, V. Zolyomi, M. Kertesz, and G. Sun, New J. Phys. 5, 125 (2003).

${ }^{44}$ M. H. F. Sluiter and Y. Kawazoe, Phys. Rev. B 69, 224111 (2004).

${ }^{45}$ G. Sun, J. Kürti, M. Kertesz, and R. H. Baughman, J. Phys. Chem. B 107, 6924 (2003).

${ }^{46}$ G. Kresse and J. Furthmüller, Phys. Rev. B 54, 11169 (1996), and references therein.

${ }^{47}$ M. Hasegawa and K. Nishidate, Phys. Rev. B 70, 205431 (2004).

${ }^{48}$ A. J. Stone, The Theory of Intermolecular Forces (Oxford University Press, New York, 1996).

${ }^{49}$ P. Vinet, J. H. Rose, J. Ferrante, and J. R. Smith, J. Phys.: Condens. Matter 1, 1941 (1989).

${ }^{50}$ Q. Wu and W. Yang, J. Chem. Phys. 116, 515 (2002).

${ }^{51}$ R. Zacharia, H. Ulbricht, and T. Hertel, Phys. Rev. B 69, 155406 (2004). 